\title{
Monte Carlo analyses of blanket neutronics experiments at FNS with latest nuclear data libraries
}

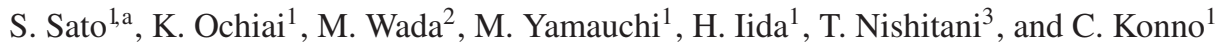 \\ 1 Japan Atomic Energy Agency, Tokai 319-1195, Japan \\ 2 Japan Computer System, Mito 310-0805, Japan \\ 3 Japan Atomic Energy Agency, Naka 311-0193, Japan
}

\begin{abstract}
A series of neutronics experiments with mockups relevant to solid breeder water cooled blanket at FNS/JAEA have been analyzed with MCNP-4C, FENDL-2.1 and JENDL-3.3. Divergences of calculation results to experimental ones (C/Es) on tritium production rates (TPRs) from unity increase in the experiment with a neutron reflector made of SS316. These also increase at boundary between the breeder and beryllium layers. This suggests that there are some problems in back-scattering cross section data of nuclei included in SS316 and beryllium. The divergence can be improved by installation of water at this boundary. The C/Es on integrated TPRs are within $7 \%$ in experiments without a reflector.
\end{abstract}

\section{Introduction}

A tritium breeding ratio (TBR) is around 1.1 for the present DEMO reactor design being developed by Japan Atomic Energy Agency (JAEA), therefore a prediction uncertainty of a TBR is required to be less than $10 \%$ to guarantee a TBR of more than unity [1]. A series of neutronics experiments have been performed at Fusion Neutronics Source (FNS) facility in JAEA with partial mockups relevant to solid breeder water cooled test blanket module being developed by JAEA for installation in ITER [2]. Tritium production rates (TPRs) have been measured in the blanket mockups. Mainly three experiments have been done. (1) Beryllium and enriched breeder mockup [3]; Calculation accuracy on TPR has been mainly evaluated on beryllium and enriched $\mathrm{Li}_{2} \mathrm{TiO}_{3}$. In addition, impacts of a reflector have been evaluated on calculation accuracy. (2) Pebble bed mockup [4]; Calculation accuracy has been evaluated for pebble bed layer. (3) Mockup with water panels [4]; Calculation accuracy has been evaluated taking into account effects of water and F82H. In the present study, these experiments have been analyzed using Monte Carlo code MCNP-4C [5] with the latest nuclear data libraries FENDL-2.1 [6] and JENDL-3.3 [7] to evaluate prediction accuracy of TPR. Calculation accuracies have been compared among three experiments, and concerns have been clarified.

\section{Overview of experiments}

\subsection{Beryllium and enriched breeder mockup}

Experiments have been performed under conditions with and without a reflector surrounding a DT neutron source. Figure 1 shows the experimental assembly with a reflector. The mockup is composed of $16 \mathrm{~mm}$ thick F82H, $12 \mathrm{~mm}$ thick enriched $\mathrm{Li}_{2} \mathrm{TiO}_{3}$ ( ${ }^{6} \mathrm{Li}$ enrichment of $40 \%$ ) and $203.2 \mathrm{~mm}$ thick beryllium. A reflector is made of SS316. Enriched

\footnotetext{
${ }^{a}$ Presenting author, e-mail: sato.satoshi92@jaea.go.jp
}

$\mathrm{Li}_{2} \mathrm{CO}_{3}$ pellets are applied as TPR detectors. Dimensions of pellets are $13 \mathrm{~mm}$ in diameter and $0.5-2 \mathrm{~mm}$ in thickness. 15 pellets with total thickness of $12 \mathrm{~mm}$ have been installed at the center of $\mathrm{Li}_{2} \mathrm{TiO}_{3}$ layer. Tritium activities produced in these irradiated pellets are measured with a liquid scintillation counter (LSC) after wet-chemistry treatment procedure, thus evaluating detailed distribution of TPRs.

\subsection{Pebble bed mockup}

This experiment has been performed under a condition without a reflector. Figure 2 shows the mockup. The mockup is composed of $15 \mathrm{~mm}$ thick $\mathrm{Li}_{2} \mathrm{O}$ (natural enrichment) pebble bed layer with $1.8 \mathrm{~mm}$ thick F82H and $101.6 \mathrm{~mm}$ thick beryllium block layers. The pebble diameter is $1 \mathrm{~mm}$ and the packing fraction is $58 \%$. In order to measure a detailed spatial

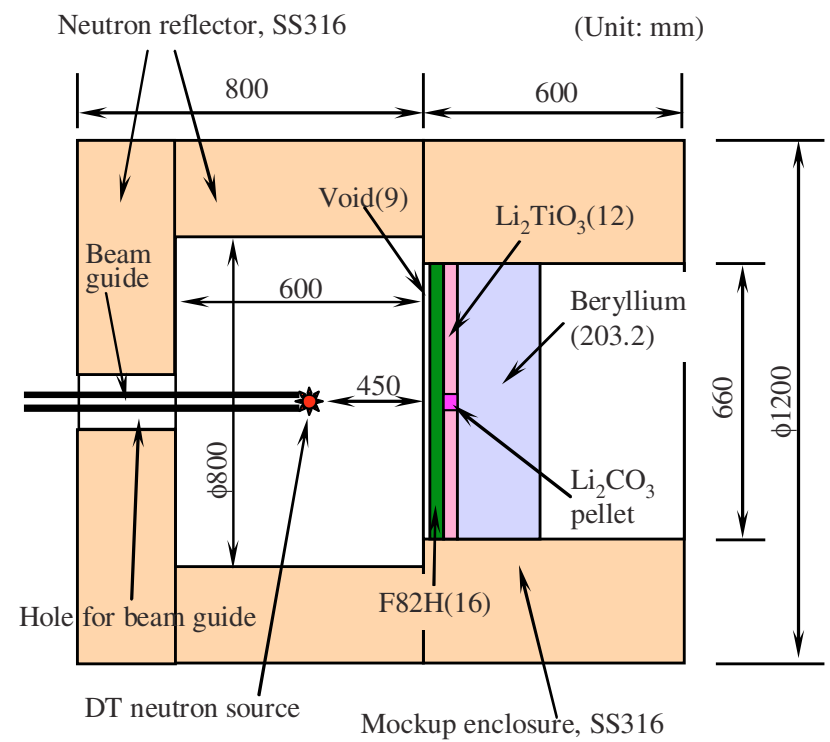

Fig. 1. Experimental assembly with a neutron reflector. 


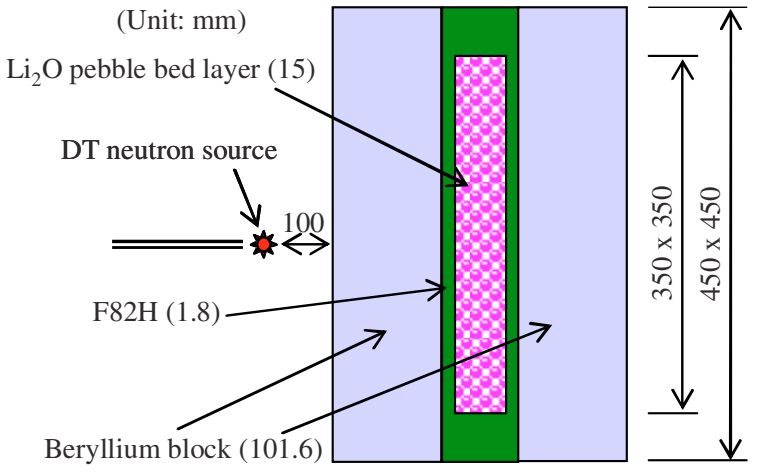

Fig. 2. Pebble bed mockup.

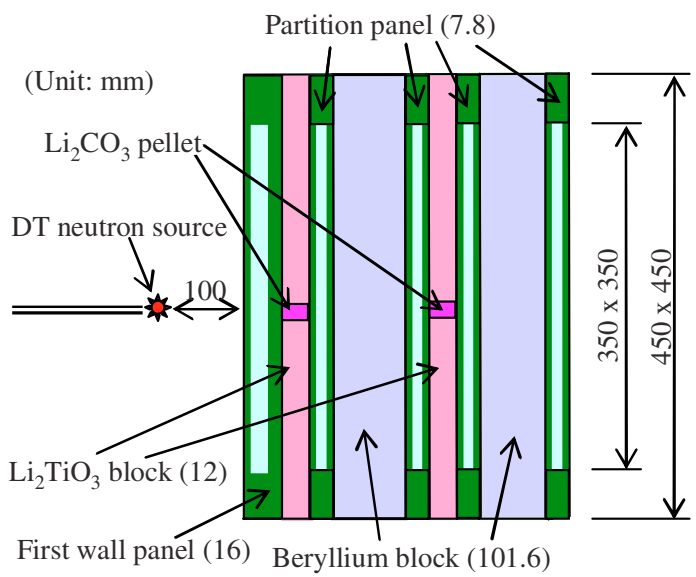

Fig. 3. Mockup with water panels.

distribution of TPR in the pebble bed layer, pebble bed detectors are applied for measurement of TPR. An aluminum cylinder with a thin wall ( $0.1 \mathrm{~mm}$ thickness) filled with pebbles and sectioned on eight equal parts with a diameter of $13 \mathrm{~mm}$ and a width of $1.85 \mathrm{~mm}$ was installed at the center of the pebble bed layer as a TPR detector. Tritium activities produced in the pebbles are measured with an LSC.

\subsection{Mockup with water panels}

This experiment has been also performed under a condition without a reflector. Figure 3 shows the mockup. The mockup is composed of $16 \mathrm{~mm}$ thick first wall panel, $12 \mathrm{~mm}$ thick enriched $\mathrm{Li}_{2} \mathrm{TiO}_{3}\left({ }^{6} \mathrm{Li}\right.$ enrichment of $40 \%$ ) layers, $101.6 \mathrm{~mm}$ thick-beryllium layers and $7.8 \mathrm{~mm}$ thick-partition panels. The first wall and partition panels are composed of $\mathrm{F} 82 \mathrm{H}$ and water. Thicknesses of the water are 6 and $4.2 \mathrm{~mm}$ in the first wall and partition panels, respectively.

\section{Calculation}

Experiment analyses have been performed by Monte Carlo calculation using MCNP-4C with FENDL-2.1 and JENDL3.3. Calculations for the pebble bed mockup experiment have been performed using the heterogeneous model simulating each pebble and void among adjacent pebbles with hexagonal close-packed geometry [3].

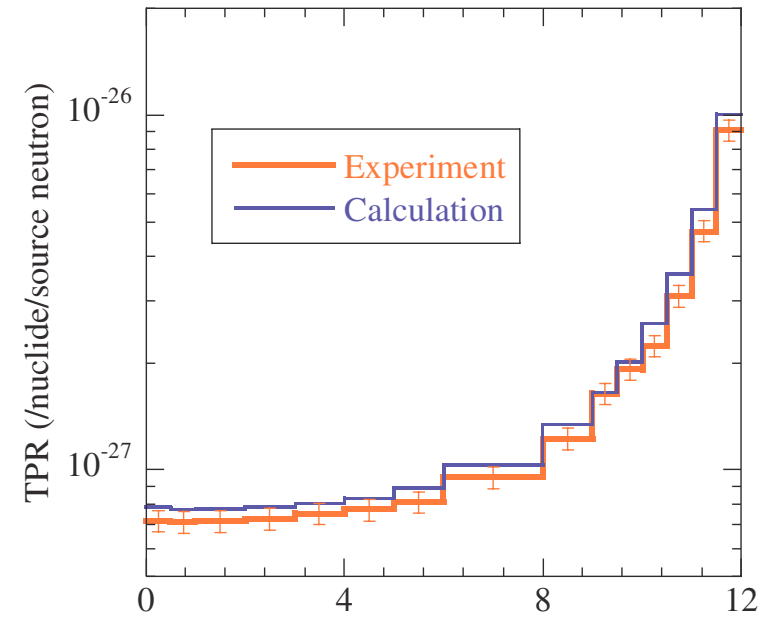

Distance from $\mathrm{Li}_{2} \mathrm{TiO}_{3}$ layer front surface $(\mathrm{mm})$

Fig. 4. Experimental and calculation results of TPR in the mockup with the neutron reflector.

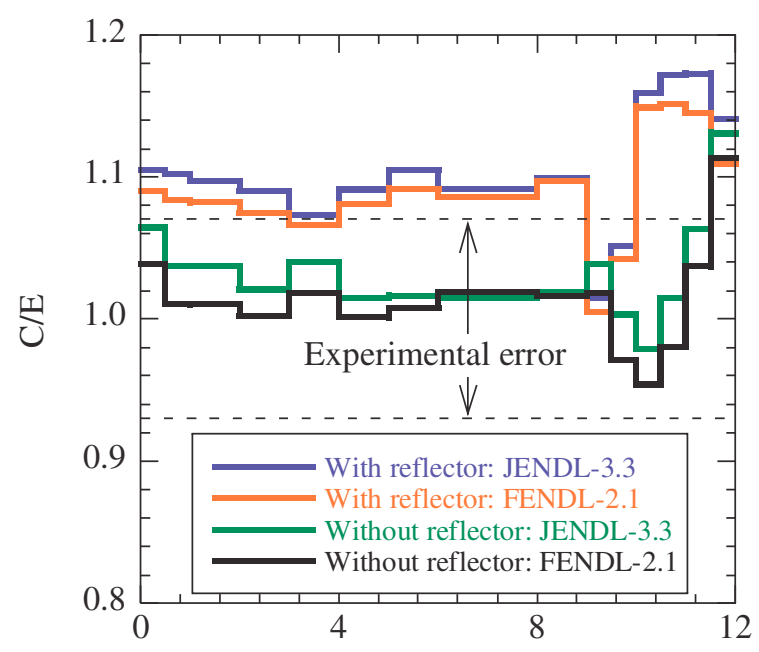

Distance from $\mathrm{Li}_{2} \mathrm{TiO}_{3}$ layer front surface (mm)

Fig. 5. $\mathrm{C} /$ Es of the TPRs with and without the reflector.

\section{Results and discussions}

\subsection{Beryllium and enriched breeder mockup}

Figure 4 shows experimental and calculation results of the TPRs on the beryllium and enriched breeder mockup with a neutron reflector by FENDL-2.1. The TPRs sharply increase with depth of $\mathrm{Li}_{2} \mathrm{TiO}_{3}$ layer due to increase of slow neutron generated by beryllium. Figure 5 shows ratio of the calculation results to the experimental ones $(\mathrm{C} / \mathrm{Es})$ for TPR with and without the neutron reflector by FENDL-2.1 and JENDL-3.3. There is no significant difference between the results by FENDL-2.1 and those by JENDL-3.3. Most of the calculation results agree well with the experimental ones within the experimental error of $7 \%$ in the mockup without the neutron reflector. On the other hand, most of calculation results overestimate experimental ones by more than $7 \%$ in the mockup with the neutron reflector. It is considered that the 
back-scattering cross section data of nuclide in SS316 have some problems.

Table 1 shows experimental and calculation results, and $\mathrm{C} / \mathrm{E}$ values of the tritium productions integrated over the diagnostic pellets, which effectively correspond to TBR, in the mockup without and with the neutron reflector. Calculation results agree well with the experimental ones within 4 and $6 \%$ for FENDL-2.1 and JENDL-3.3, respectively, in the mockup without the neutron reflector. Prediction accuracy by Monte Carlo calculation with FENDL-2.1 and JENDL-3.3 can satisfy the design target of $10 \%$. Calculation accuracy in the mockup with the neutron reflector is worse by more than $6 \%$, and cannot satisfy the design target.

Table 1. Experimental and calculation results, and $\mathrm{C} / \mathrm{E}$ values of the tritium productions integrated over the diagnostic pellets in the mockup without and with the neutron reflector.

\begin{tabular}{lll}
\hline & $\begin{array}{l}\text { Without } \\
\text { reflector }\end{array}$ & $\begin{array}{l}\text { With } \\
\text { reflector }\end{array}$ \\
\hline Experiment $^{1)}$ & $1.43 \times 10^{-5}$ & $3.05 \times 10^{-5}$ \\
Calculation $^{1)}$; FENDL-2.1 & $1.48 \times 10^{-5}$ & $3.36 \times 10^{-5}$ \\
Calculation $^{1)}$; JENDL-3.3 & $1.51 \times 10^{-5}$ & $3.42 \times 10^{-5}$ \\
C/E; FENDL-2.1 & 1.035 & 1.102 \\
C/E; JENDL-3.3 & 1.055 & 1.120 \\
\hline
\end{tabular}

1) unit: $\mathrm{Bq} /$ source neutron.

\subsection{Pebble bed mockup}

Figure 6 shows the C/E of TPRs on the pebble bed mockup experiment. The $\mathrm{C} / \mathrm{Es}$ on the tritium productions integrated over the diagnostic pebbles are 0.998 and 0.994 for FENDL2.1 and JENDL-3.3, respectively. For the pebble bed layer, calculation results also agree well with the experimental ones within $7 \%$ except for the boundary between the $\mathrm{Li}_{2} \mathrm{O}$ pebble layer and the beryllium. It can be confirmed that the TBR is accurately calculated for the pebble bed layer. On the other hand, the calculation results overestimate with depth of the breeder layer. Tritium produced by the back-scattered neutron from the rear beryllium layer increase with depth of the breeder layer. It is considered that the overestimation is due to some problems in calculation of back-scattered neutrons from the beryllium layer. This suggests that angular distributions to rear directions in the nuclear data libraries have some problems on the beryllium because there are few experimental data on the double-differential cross section to backward direction.

Table 2. Experimental and calculation results, and C/Es of the tritium productions integrated over the diagnostic pellets in the mockup with water panels.

\begin{tabular}{llll}
\hline & $1^{\text {st }}$ layer & $2^{\text {nd }}$ layer & total \\
\hline Experiment $\left.^{1}\right)$ & $5.75 \times 10^{-5}$ & $7.71 \times 10^{-5}$ & $1.35 \times 10^{-4}$ \\
\hline Cal. $^{1}$ ); FENDL-2.1 & $5.66 \times 10^{-5}$ & $7.90 \times 10^{-5}$ & $1.36 \times 10^{-4}$ \\
Cal. $^{1}$ ) JENDL-3.3 & $5.72 \times 10^{-5}$ & $8.04 \times 10^{-5}$ & $1.38 \times 10^{-4}$ \\
C/E; FENDL-2.1 & 0.984 & 1.024 & 1.007 \\
C/E; JENDL-3.3 & 0.995 & 1.043 & 1.022 \\
\hline
\end{tabular}

1) unit: $\mathrm{Bq} /$ source neutron.

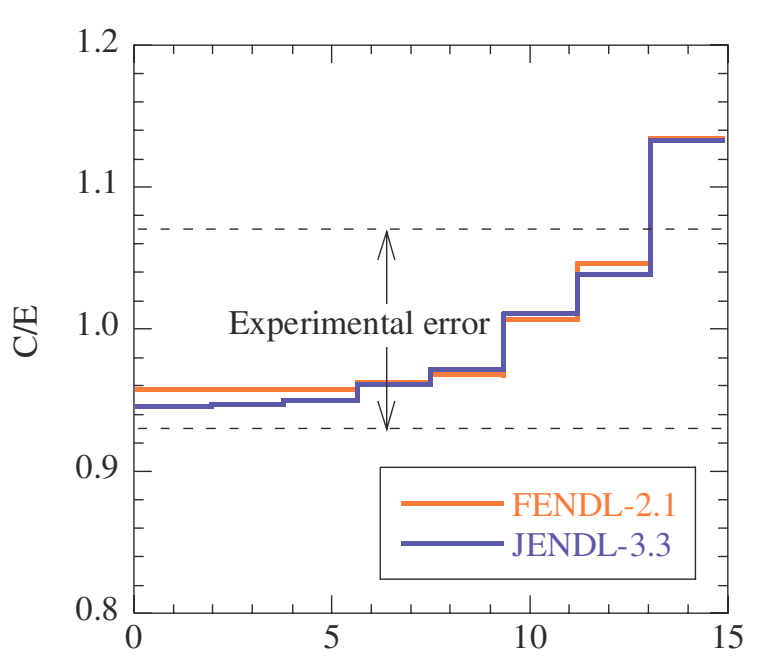

Distance from $\mathrm{Li}_{2} \mathrm{O}$ layer front surface $(\mathrm{mm})$

Fig. 6. C/Es of the TPRs on the pebble bed mockup.

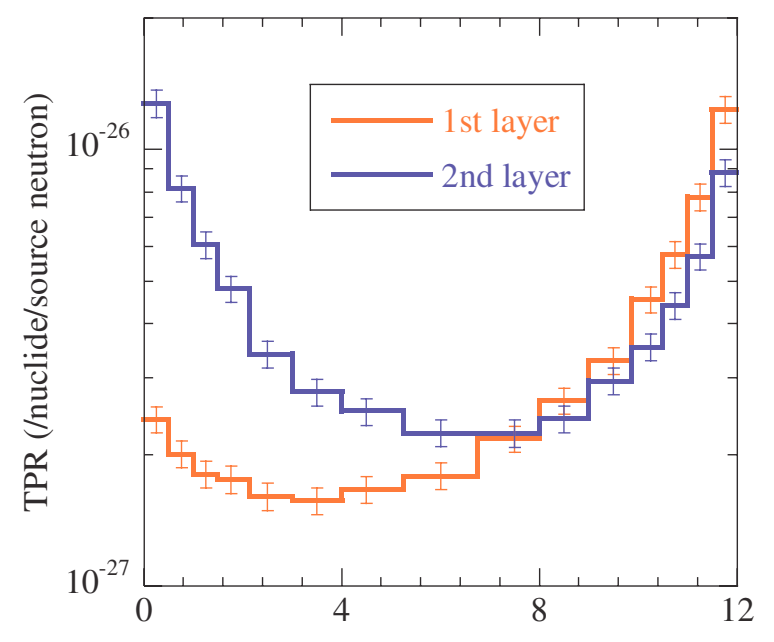

Distance from $\mathrm{Li}_{2} \mathrm{TiO}_{3}$ layer front surface (mm)

Fig. 7. Measured TPR distributions in the mockup with water panels.

\subsection{Mockup with water panels}

Figure 7 shows experimental results of TPRs in the first and second layers of the mockup with water panel. The TPRs sharply increase with decrease in distance to the beryllium layer by about one order of magnitude, and these also increase with decrease in distance to the first wall panel by a factor of about two due to moderation of fast neutron and increase of slow neutron by water. Figure 8 shows the C/E for FENDL2.1 and JENDL-3.3. Most of the calculation results agree well with the experimental ones within the experimental error of $7 \%$. Calculation results also agree well with the experimental ones within $7 \%$ at the boundary between $\mathrm{Li}_{2} \mathrm{TiO}_{3}$ and beryllium. In this mockup, the partition panels with water are installed at the boundary. Hydrogen in the water makes many thermal neutrons, and may eliminate the overestimation due to beryllium at the boundary between $\mathrm{Li}_{2} \mathrm{TiO}_{3}$ and beryllium. Table 2 shows experimental and calculation results, and C/Es of the tritium productions integrated over the diagnostic 


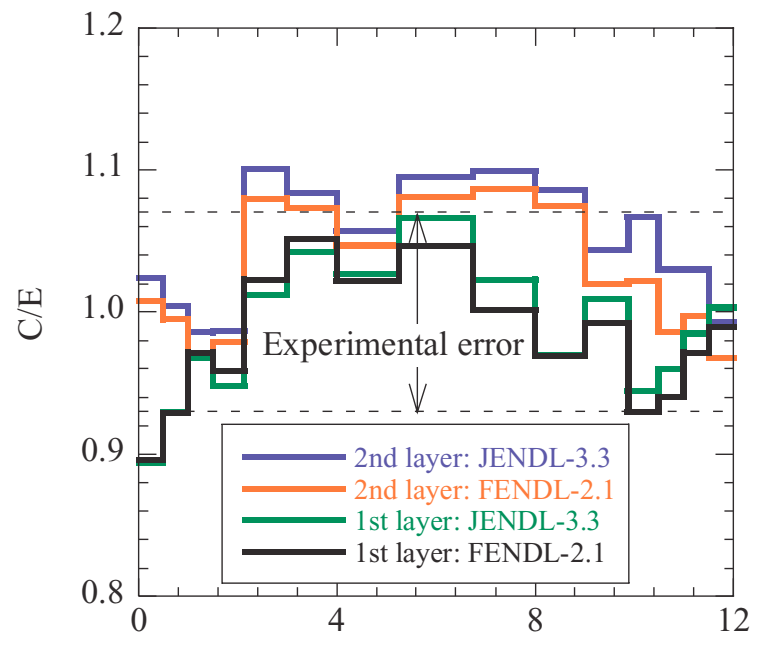

Distance from $\mathrm{Li}_{2} \mathrm{TiO}_{3}$ layer front surface $(\mathrm{mm})$

Fig. 8. $\mathrm{C} / \mathrm{E}$ of the TPRs.

pellets. For the TBR in the first and second layers, prediction accuracy can fully satisfy design target of $10 \%$.

\section{Summary}

Analyses have been performed on a series of blanket neutronics experiments at JAEA FNS using MCNP-4C with the latest nuclear data libraries FENDL-2.1 and JENDL-3.3, 1) Beryllium and enriched breeder mockup, 2) Pebble bed mockup, 3) Mockup with water panel. From the present study, the following findings have been obtained.
1) In all the experiments without a neutron reflector, the calculation results of the tritium production integrated over the detectors agree well with the experimental ones within experimental error of $7 \%$.

2) In the experiment with a neutron reflector, the calculation results overestimate the experimental ones by more than $10 \%$. This suggests that there are some problems on in back-scattering cross section data of nuclei included in SS316.

3) The calculation results of local tritium production rates at boundary between the breeder and beryllium layers also overestimate the experimental ones by more than $10 \%$. On the other hand, this overestimation can be eliminated by installation of water at the boundary.

The authors would like to thank C. Kutsukake, S. Tanaka, Y. Abe, M. Seki, Y. Oginuma and M. Kawabe for operation of the FNS accelerator. The authors thank Drs M. Seki, S. Seki and H. Takatsu for their support and encouragement.

\section{References}

1. M. Enoeda et al., Nucl. Fus. 43, 1837 (2003).

2. M. Enoeda et al., Fus. Eng. Des. 81, 415 (2006).

3. S. Sato et al., Fus. Sci. Technol. 47, 1046 (2005).

4. S. Sato et al., Nucl. Fus. (to be published).

5. J.F. Briesmeister (ed.), Los Alamos National Laboratory Report LA-13709-M, 2000.

6. D. Lopez Al-dama, A. Trkov, IAEA Report INDC(NDS)-467, 2004.

7. K. Shibata et al., J. Nucl. Sci. Technol. 39, 1125 (2002). 